\title{
Effects of Working Memory Load on Frontal Connectivity in Children with Autism Spectrum Disorder: an fNIRS study
}

Yvonne M.Y. Han ( $\nabla$ yvonne.han@polyu.edu.hk )

The Hong Kong Polytechnic University

Ming-Chung Chan

The Hong Kong Polytechnic University

Melody M.Y. Chan

The Hong Kong Polytechnic University

Agnes S Chan

Chinese University of Hong Kong

Michael K Yeung

The Hong Kong Polytechnic University

\section{Research Article}

Keywords: ASD, working memory, functional connectivity, frontal lobe, near-infrared spectroscopy

Posted Date: August 31st, 2021

DOI: https://doi.org/10.21203/rs.3.rs-855822/v1

License: (c) (i) This work is licensed under a Creative Commons Attribution 4.0 International License. Read Full License

Version of Record: A version of this preprint was published at Scientific Reports on January 27th, 2022.

See the published version at https://doi.org/10.1038/s41598-022-05432-3. 


\section{Effects of Working Memory Load on Frontal Connectivity in}

\section{Children with Autism Spectrum Disorder: an fNIRS study}

Yvonne M Y Han ${ }^{1,2^{*}}$; Ming-Chung Chan ${ }^{1}$; Melody M Y Chan ${ }^{1}$; Agnes S Chan ${ }^{3}$; Michael K Yeung ${ }^{1,2}$

${ }^{1}$ Department of Rehabilitation Sciences, The Hong Kong Polytechnic University, Hong Kong SAR

${ }^{2}$ University Research Facility in Behavioral and Systems Neuroscience (UBSN), The Hong Kong Polytechnic University, Hong Kong SAR

${ }^{3}$ Department of Psychology, The Chinese University of Hong Kong, Hong Kong SAR

*Corresponding Author:

Yvonne M.Y. Han, Ph.D.

Department of Rehabilitation Sciences

The Hong Kong Polytechnic University

Hung Hom, Kowloon

Hong Kong SAR.

Tel: (852) 2766-7578

Fax: (852) 2330-8656

E-mail: yvonne.han@polyu.edu.hk 


\begin{abstract}
Background: Individuals with autism spectrum disorder (ASD) perform poorly in working memory (WM) tasks, with some literature suggesting that their impaired performance is modulated by WM load. While some neuroimaging and neurophysiological studies have reported altered functional connectivity during WM processing in these individuals, it remains largely unclear whether such alterations are moderated by WM load. The present study aimed to examine the effect of WM load on functional connectivity within the prefrontal cortex (PFC) in ASD using functional near-infrared spectroscopy (fNIRS).
\end{abstract} Method: Twenty-two children with high-functioning ASD aged 8-12 years and 24 age-, intelligent quotient (IQ)-, sex- and handedness-matched typically developing (TD) children performed a number $n$-back task with three WM loads (0-back, 1-back, and 2-back). Hemodynamic changes in the bilateral lateral and medial PFC during task performance were monitored using a multichannel NIRS device. Results: Children with ASD demonstrated slower reaction times, specifically during the "low load" condition, than TD children. In addition, the ASD and TD groups exhibited differential load-dependent functional connectivity changes in the lateral and medial PFC of the right but not the left hemisphere. Conclusion: These findings indicate that WM impairment in high-functioning ASD is paralleled by load-dependent alterations in right, but not left, intrahemispheric connectivity during WM processing in children with ASD. A disruption of functional neural connections that support different cognitive processes may underlie poor performance in WM tasks in ASD.

Keywords: ASD, working memory, functional connectivity, frontal lobe, near-infrared spectroscopy 
Autism spectrum disorder (ASD) is a neurodevelopmental disorder characterized by disturbances in social communication, as well as manifestations of restricted and stereotyped behavior (American Psychiatric Association, 2013). It has been suggested that deficiencies in working memory (WM), which is the temporary storage system that serves as a basis for complex cognitive functions (Baddeley, 2012), and its associated executive functioning deficits (Demetriou et al., 2018) are fundamental to ASD (Cui, Gao, Chen, Zou, \& Wang, 2010; de Vries \& Geurts, 2014; Kleinhans, Akshoomoff, \& Delis, 2005; Lemon, Gargaro, Enticott, \& Rinehart, 2011). Many individual studies have demonstrated deficient WM in individuals with ASD, but the findings have been inconsistent: some studies have found a significant difference in WM task performance between individuals with ASD and typically developing (TD) controls (Corbett, Constantine, Hendren, Rocke, \& Ozonoff, 2009; Gabig, 2008; Sinzig, Morsch, Bruning, Schmidt, \& Lehmkuhl, 2008; Williams, Goldstein, \& Minshew, 2006), whereas others have not (Geurts, Verté, Oosterlaan, Roeyers, \& Sergeant, 2004; Happé \& Frith, 2006; Ozonoff \& Strayer, 2001). Nevertheless, a recent meta-analysis confirmed the presence of WM deficits in ASD (Habib, Harris, Pollick, \& Melville, 2019), with previous researchers suggesting that the level of WM impairment might be associated with the increase in the complexity of information and the demand for WM capacity involved in a task (Barendse et al., 2013).

It is well established that the frontal lobe is involved in WM. Evidence for this comes from studies of the $n$-back task, one of the most widely adopted paradigms reflecting WM ability in cognitive neuroscience. For instance, an aggregate of studies has shown bilateral activation in the dorsolateral and ventrolateral PFC, dorsal cingulate cortex, medial premotor cortex, and parietal cortex during the n-back task (Owen, McMillan, Laird, \& Bullmore, 2005). Additionally, functional coupling between frontal and parietal regions (Ma et al., 2012) and 
between the left and right PFC (Sun et al., 2019) increases during processing that requires increased WM demand. These studies provide evidence to support the notion that WM is mediated by the PFC and its distributed neural networks (Fletcher \& Henson, 2001; Frank, Loughry, \& O'Reilly, 2001; Smith \& Jonides, 1999), which also implies that good performance in a WM task requires an efficient flow of information between these brain areas to maintain and integrate information.

Frontal lobe dysfunction in ASD has long been documented in literature. For example, a neuroimaging meta-analysis showed that children with high-functioning ASD showed hypoactivation in the right inferior frontal gyrus and right anterior cingulate gyrus during cognitive tasks involving inhibition, switching and updating components (Zhang, Peng \& Zhang, 2020). In addition, Narita et al. (2012) reported abnormal activation patterns in ASD adults over the left dorsolateral prefrontal cortex (PFC) during a visuospatial delayed recall task, a task reflecting participants' WM capacity. These studies collectively imply that abnormal frontal activity might be associated with impaired cognitive performance in ASD.

Furthermore, converging evidence suggests altered functional connectivity of the brain involving the PFC in both resting and task states in ASD, although it is still controversial whether these alterations should be best characterized as global underconnectivity and/or local overconnectivity (Hull et al., 2017; Just, Keller, Malave, Kana, \& Varma, 2012; Mohammad-Rezazadeh, Frohlich, Loo, \& Jeste, 2016). Since functional coupling between and within regions in the brain, especially the PFC, is important for the execution of WM tasks, altered connectivity of the brain may underlie the WM deficits exhibited by individuals with ASD. Indeed, there have been some studies reporting altered functional connectivity during $n$-back tasks in ASD. Some early fMRI studies found that adults with ASD exhibited overall underconnectivity between the PFC and parietal regions during a letter 2-back task 
(Koshino et al., 2005) and between the frontal and fusiform or parietal areas across different WM load levels in a facial $n$-back task (Koshino et al., 2008). Additionally, a more recent magnetoencephalography study found reduced synchronization within frontotemporal networks during a visuospatial 2-back task in children with ASD compared to TD children (Urbain et al., 2016). Despite these findings, it remains largely unclear whether the altered functional connectivity during WM task performance in individuals with ASD is moderated by WM load. Thus, the aim of the present study was to examine the effect of WM load on functional connectivity in ASD.

Functional near-infrared spectroscopy (fNIRS) is a noninvasive neuroimaging technique that monitors changes in the concentration of oxyhemoglobin $(\mathrm{HbO})$ and deoxyhemoglobin $(\mathrm{HbR})$, which are associated with activities of the cerebral cortex (Villringer \& Chance, 1997). During fNIRS recording, an fNIRS device emits at least two near-infrared lights with a wavelength of 650-900 nm into the scalp. The lights then penetrate the scalp, skull, cerebrospinal fluid, and brain tissue in a banana-shaped trajectory, and the exiting lights are recorded by a receiver. Variations in optical density caused by changes in the brain's metabolism can then be used to estimate the changes in hemoglobin concentration in the sampled brain issue via application of the modified Beer-Lambert law (Delpy et al., 1988). Over the last decade, fNIRS has been used to assess the functional coupling of brain regions, especially the PFC, during performance of a wide variety of tasks. For example, fNIRS has been used to measure functional connectivity between two cortical areas that underlie motor performance (Varshney, Liapounova, Golestani, Goodyear, \& Dunn, 2012). It has also been used to measure intrahemispheric and/or interhemispheric connectivity within the dorsolateral PFC and frontal pole during the $n$-back task in healthy young adults (Fishburn, Norr, Medvedev, \& Vaidya, 2014; Sun et al., 2019). 
It is widely agreed that fNIRS possesses adequate temporal and spatial resolution and relatively high tolerance to motion and environmental conditions (Boas, Dale, \& Franceschini, 2004). These unique properties make fNIRS a suitable tool for studying functional connectivity in pediatric populations. In the present study, we utilized fNIRS to examine functional connectivity within the PFC during the $n$-back task with three WM loads in children with high-functioning ASD. We hypothesized that compared to TD controls, the children with ASD would exhibit WM impairment and altered PFC connectivity that is loaddependent during $n$-back task performance.

\section{Method}

\section{Participants}

This study was conducted in accordance with the Declaration of Helsinki. The research protocol was approved by the Human Subjects Ethics Sub-Committee of Hong Kong Polytechnic University. Twenty-two children with ASD and 24 TD children aged 8-12 years voluntarily participated in this study. These children were recruited through advertisements sent to parent groups via social media (e.g., WhatsApp and Facebook) and posted in the rehabilitation clinic at Hong Kong Polytechnic University, as well as through invitation letters sent to teachers of local primary schools. All potential participants were screened for eligibility prior to the study, and those with a history of epilepsy or head trauma were excluded. A participant was included in the high-functioning ASD group if he/she fulfilled the following criteria: 1) he/she had received a diagnosis of ASD based on the Diagnostic and Statistical Manual of Mental Disorders $-5^{\text {th }}$ Edition (DSM-5; APA, 2013) from psychiatrists before the commencement of this study and scored above the cutoff points in all subscales in 
the Autism Diagnostic Interview-Revised (ADI-R; Lord et al., 1994) that was conducted by a registered clinical psychologist during the screening session for this study and 2) obtained a full-scale intelligent quotient (IQ) $\geq 80$ on the Hong Kong version of the Wechsler Intelligence Scale for Children-Fourth Edition short form [WISC-IV (HK); Wechsler, 2003], which was also administered by the clinical psychologist during the screening session. For a participant to be included in the TD group, he/she should have no history of developmental delay or any other neurological/psychiatric disorders.

\section{Procedure and materials}

All children and their parents attended individual screening and neuropsychological/ neurophysiological measurement sessions at The Hong Kong Polytechnic University. Prior to the screening and assessment, the children and their parents were informed about the assessment procedures, and informed assent and consent were obtained from the children and parents. All of the child participants were administered the Tumbling "E" Test to ensure that they had normal or corrected-to-normal vision before any cognitive assessment and fNIRS testing. The child participants were screened for intellectual functioning by a clinical psychologist, followed by fNIRS recording sessions conducted by trained research assistants. These procedures were conducted in separate rooms. Short breaks were given to the participants every 30 minutes, and the entire experiment lasted for approximately two hours for each participant.

The demographic information of the participants was collected during interviews conducted by a clinical psychologist with parents in a separate room. To measure participants' level of autistic traits in both the ASD and TD groups, the Social Responsiveness Scale-Second Edition (SRS-2) was administered (Constantino \& Gruber, 2012). The SRS-2 is a sensitive measure of children's social impairment related to ASD over the past six months. It consists 
of five subscales that measure social awareness, social cognition, social communication, social motivation, and restricted interests and repetitive behavior. Each item is rated on a four-point Likert scale from 1 (not true) to 4 (almost always true). Higher total scores indicate greater difficulties in socialization. For the assessment of IQ,WISC-IV (HK) short form was adopted in this study, which comprises two verbal subtests, digit span and similarities, and two performance subtests, matrix reasoning and coding, yielding a full IQ score with a mean of 100 and a standard deviation of 15.

\section{$N$-back paradigm}

Each participant performed the $n$-back task, which was run by E-prime 2.0 Software (Psychology Software Tools, Pittsburgh, PA, USA), while prefrontal hemodynamic changes were recorded by fNIRS. The $n$-back task was adapted from previous fNIRS studies (Ehlis, Bähne, Jacob, Herrmann, \& Fallgatter, 2008; Yeung, Lee, \& Chan, 2019; Yeung et al., 2016) and consisted of the 0-back (i.e., low WM load), 1-back (i.e., medium WM load), and 2-back (i.e., high WM load) conditions (Figure 1). Each condition was presented twice, and the three tasks alternated in blocks (i.e., 0-1-2-0-1-2, 0-2-1-0-2-1, 1-0-2-1-0-2, 1-2-0-1-2-0, 2-0-1-2-01 or $2-1-0-2-1-0)$ and were separated by 30 -second rest blocks during which the participants sat still with their eyes open. The order was counterbalanced across participants to prevent order effects. Each task block consisted of a 5-second instruction cue that introduced the task, followed by 20 trials ( 5 target and 15 non-target trials) presented in a pseudorandom manner.

In the 0 -back condition, the participants were instructed to press the left button of a mouse when the digit " 0 " (i.e., target) appeared but to press the right button for other digits. In the 1back condition, the participants were instructed to press the left button when the presented digit was identical to the digit presented in the previous trial (i.e., target) or otherwise, the right button. In the 2-back condition, the participants were instructed to press the left button 
when the presented digit was identical to the digit presented two trials before (i.e., target) or otherwise, the right button. Each digit was presented for 500 milliseconds, followed by an inter-stimulus interval of 1500 milliseconds. Each task block lasted for 45 seconds, and the total duration was 8 minutes.

\section{fNIRS measurement}

The fNIRS recording session was conducted in a quiet, dim-lighted room (Orihuela-Espina, Leff, James, Darzi, \& Yang, 2010). Each participant sat $60 \mathrm{~cm}$ away from a 15" LCD monitor that was used for stimulus display. Their head dimensions (nasion-inion and left-right periauricular points) were measured to facilitate offline spatial registration of fNIRS channels (Singh, Okamoto, Dan, Jurcak, \& Dan, 2005) where the channel positions were transformed into the Montreal Neurological Institute (MNI) space and projected onto the surface of a volume rendered children brain template chosen according to participants' age and head dimensions (Sanchez, Richards, \& Almli, 2012).

Prefrontal hemodynamics during the $n$-back task were measured by a 52-channel fNIRS optical topography system (ETG-4000; Hitachi Medical Co., Tokyo, Japan). It consisted of 17 sources of two wavelengths (695 and $830 \mathrm{~nm})$ and 16 detectors. A custom-built headband mounted with optical emitters and receivers, which were arranged in a $3 \times 11$ matrix, was placed on the children's heads based on the 10-10 system (Jurcak, Tsuzuki, \& Dan, 2007), an extension to the international 10-20 system (Jasper, 1958). The probe in the center of the lowest row was placed at Fpz, guided by a reference point marked on the headband that was standardized to be placed over the nasion of all subjects. The sampling frequency was $10 \mathrm{~Hz}$. Each pair of sources and detectors was $3 \mathrm{~cm}$ apart, and therefore, measured brain activity approximately 15-20 mm beneath the scalp (Cui, Bray, Bryant, Glover, \& Reiss, 2011). 


\section{Statistical analysis}

Behavioral data. The mean latency time for all correct trials for each subject was calculated and named the mean reaction time (RT), and the percentage of correct trials as accuracy (ACC) for the $n$-back task was analyzed. We first inspected normality of the RT and ACC distributions with Shapiro-Wilk tests. Because RT variables in all three conditions were positively skewed, they were log-transformed. Given that most log-transformed RT variables no longer violated the normal distribution, $p \mathrm{~s}>0.05$, parametric tests were used for analysis. For each $n$-back condition, ACC and mean RT were separately analyzed with two-way mixed-design analysis of variance (ANOVA) with load (low, medium, and high) as the within-subjects factor and group (ASD and TD) as the between-subjects factor.

fNIRS processing. We performed functional connectivity followed by activation analyses. For both analyses, the raw fNIRS signals were first converted to optical density changes and then transformed into changes in $\mathrm{HbO}$ and $\mathrm{HbR}$ using the modified BeerLambert law (Delpy et al., 1988). Since HbO has a higher signal-to-noise ratio than HbR (Cui et al., 2011; Kamran, Mannan, \& Jeong, 2016; Strangman, Culver, Thompson, \& Boas, 2002), only the HbO data were analyzed. While we focused on the lateral PFC because this region has been implicated in $n$-back task performance (Owen et al., 2005), the medial frontopolar region was also examined to assess the specificity of the results. Based on the virtual registration of channels (Singh et al., 2005), eight and five channels represented the lateral PFC and medial frontopolar cortex on each side, respectively (Figure 2). Channels 7, $8,18,28,29,39,49$, and 50 represented the left lateral PFC; channels 3, 4, 14, 24, 25, 35, 45, and 46 represented the right lateral PFC; channels $6,17,27,38$, and 48 represented the left medial PFC; and channels 5, 15, 26, 36, and 47 represented the right medial PFC. The 
outermost channels covering the temporal lobe regions were not analyzed because of poor optode-scalp contact for most of the children.

Functional connectivity analysis. The connectivity patterns during the $n$-back task were analyzed using the NIRS Brain AnalyzIR Toolbox (Santosa, Zhai, Fishburn, \& Huppert, 2018) for MATLAB (The Mathworks, Natick, MA). The traditional approach to connectivity analysis computes the Pearson correlations between possible pairs of channels, but this approach did not address systemic physiological noise and head motion, which are temporally correlated (colored) noise in the fNIRS signals and may affect the accuracy of the results (Santosa et al., 2017). In this case, a prewhitening filter can be used to remove the autocorrelation and whiten the frequency content of the signal (Barker, Aarabi, \& Huppert, 2013). First, temporally correlated physiological artifacts were corrected for by applying an autoregressive prewhitening filter to the signal $(Y)$ received by each channel, defined as $Y_{\{t\}}=\sum_{i=1}^{P} a_{i} \cdot Y_{\{t-i\}}+\epsilon_{\{t\}}$ and $\epsilon_{\{t\}} \in N\left(0, \sigma^{2}\right)$, where $\mathrm{t}$ indicates the sample point, the set $a_{i}$ is the autoregressive coefficient of the model, and $\mathrm{P}$ is the model order that was selected based on Bayesian information criteria (BIC). This equation states that the current sample point $(\mathrm{Y}\{\mathrm{t}\})$ can be predicted based on the last several time points (a1 . $\mathrm{Y}\{\mathrm{t}-1\} \ldots$ ap .Y $\{t-p\})$ and newly added information at that time point, which are the innovations and denoted as $(\varepsilon\{t\})$. The innovations can be thought of as new information that is added to the total signal at each time point. This method yielded prewhitened signals without autocorrelations with time (Barker, Aarabi, \& Huppert, 2013; Huppert, 2016; Santosa et al., 2018). The prewhitened signals were then processed using the procedure described in Santosa et al. (2017) to remove motion artifacts. Then, correlation coefficients $(R)$ between two prewhitened and preweighted signals (denoted as $A_{S, W}$ and $B_{S, W}$ below) were calculated using the equation $R=b_{1} \cdot \frac{\sigma_{A}}{\sigma_{B}}$ (where $\sigma_{A}$ and $\sigma_{B}$ are the standard deviations of the signals $\mathrm{A}$ and $\mathrm{B}$ ) 
after obtaining the value $b_{1}$ estimated by applying a least-square solution to the regression model $A_{S, W}=b_{0}+B_{S, W} \cdot b_{1}$. This process was repeated until the correlation coefficients of all possible channel pairs were calculated. The correlation coefficients for each channel pair were Fisher's $Z$-transformed before averaging across channel pairs.

Intrahemispheric and interhemispheric connectivity were examined separately for the lateral and medial PFC. The channel pairs for each connectivity measure are presented in Figure 3. First, a mixed ANOVA with load (low, medium, and high), region (medial and lateral) and hemisphere (left and right) as within-subjects factors and group (ASD and TD) as a betweensubjects factor was conducted on the mean Z-transformed correlation coefficients for intrahemispheric connectivity. Then, another mixed ANOVA with load and region as withinsubjects factors and group as a between-subjects factor was conducted on the mean Ztransformed correlation coefficients for interhemispheric connectivity.

Activation analysis. To complement the connectivity analysis, task-related changes in $\mathrm{HbO}$ were also examined. The fNIRS signals between each source and detector pair were analyzed using a general linear regression model (GLM) to test for differences between the baseline (30-s rest period at the beginning of the experiment) and the task condition. This can be expressed by the equation $\mathrm{Y}=\mathrm{X} \times \beta+\mathrm{e}$, where $\mathrm{X}$ is the timing of stimulus events, $\beta$ is the coefficient (weight) of the stimulus condition, and $\mathrm{Y}$ is the vector of measurements. Several modules can be used to obtain $\beta$, and the AR-IRIS method is the one we used in the paper; this method can remove artifacts and control type-I errors in the fNIRS model (see Santosa et al., 2018 for details). These values were then averaged across repetitions for each WM load and across channels for the lateral and medial PFC separately. For each region, a mixed ANOVA with load, hemisphere and region as within-subjects factors and group as a betweensubjects factor was conducted on $\beta$. 
For significant interactions and main effects of group in the behavioral and neurophysiological measures, if any, post hoc independent-/paired-sample $t$-tests with Bonferroni corrections for three WM-load conditions (i.e., $\mathrm{p}<.017$ for each condition) were conducted.

\section{Results}

\section{Sample characteristics}

Table 1 shows the demographic, intellectual, and clinical characteristics of the participants. Independent-sample $t$-tests and chi-square tests showed that the TD and ASD groups did not significantly differ in age $(p=.93)$, IQ $(\mathrm{p}=.22)$ or sex $(\mathrm{p}=.20)$. Regarding autism symptomatology indicated by the SRS-2, the children with ASD had a significantly higher SRS-2 total score than the TD individuals $(\mathrm{p}<.001)$. Furthermore, there were no significant differences based on handedness in any of the $n$-back behavioral and fNIRS measures in the TD group $(p s>.05)$.

\section{Behavioral performance}

Table 2 presents the ACC and mean (log-transformed) RT in each group. For the ACC measure, a mixed $3($ load $) \times 2$ (group) ANOVA showed a significant main effect of load $\left(F_{2,88}\right.$ $\left.=69.71, p<.001, \eta_{p}^{2}=.61\right)$. The main effect of group was not significant $\left(F_{1,44}=1.87, p\right.$ $=.18, \eta_{p}^{2}=.04$ ), and no significant load $\mathrm{x}$ group interaction effect was observed.

For the mean log-transformed RT measure, a 3 (load) $\times 2$ (group) mixed ANOVA showed significant main effects of load $\left(F_{2,88}=35.84, p<.001, \eta_{p}^{2}=.45\right)$ and group $\left(F_{1,44}=6.53, p\right.$ 
$=.014, \eta_{p}^{2}=.13$ ), with the ASD group having a slower mean log-transformed RT than the TD group. The interaction between group and load was not significant $\left(F_{2,88}=0.33, p=.72, \eta_{p}^{2}=\right.$ .008). Post hoc $t$-tests with Bonferroni corrections showed that the ASD group had significantly slower mean log-transformed RT than the TD group in the 0-back condition ( $p$ $=.009)$ and trends toward significance in the 1-back $(p=.030)$ and 2-back $(p=.025)$ conditions that did not survive Bonferroni corrections.

\section{Effect of n-back load on prefrontal functional connectivity}

To clarify the neural mechanisms underlying $n$-back task performance, we analyzed connectivity within and between the left and right medial and lateral PFC (Table 3), which were the regions of interest (ROIs) defined in our study (Figure 4). Regarding intrahemispheric connectivity, a 2 (hemisphere) $\times 3$ (load) $\times 2$ (region) $\times 2$ (group) mixed ANOVA showed a significant hemisphere $\times$ load $\times$ region $\times$ group effect $\left(F_{2,88}=4.478, p\right.$ $\left.=.014, \eta_{p}^{2}=.09\right)$. The main effects were significant for hemisphere $\left(F_{1,44}=4.350, p=.043\right.$, $\left.\eta_{p}^{2}=.09\right)$ and region $\left(F_{1,44}=4.194, p=.047 \eta_{p}^{2}=.09\right)$. A follow-up $3($ load $) \times 2($ region $) \times 2$ (group) mixed ANOVA was conducted for each hemisphere to explore how different loads in the n-back task mediated functional connectivity of the medial and lateral PFC on each side of the brain. In the right hemisphere, a significant load $\times$ region $\times$ group interaction $\left(F_{2,88}=\right.$ $\left.6.073, p=.003, \eta_{p}^{2}=.12\right)$ and a significant main effect of region $\left(F_{1,44}=7.351, p=.010\right.$, $\left.\eta_{p}^{2}=.14\right)$ were found. Thus, the ASD and TD groups had different connectivity changes in the right hemisphere with load in the lateral and medial PFC. A post hoc paired-sample t-test showed a trend toward significance between 0-back and 1-back connectivity in the right medial regions of the ASD group $(\mathrm{p}=.030)$, which did not survive Bonferroni correction. On 
the left side, neither a significant main effect nor a significant interaction was found (ps $>.24)$.

Regarding interhemispheric connectivity, a 3 (load) $\times 2$ (region) $\times 2$ (group) mixed ANOVA showed that only the main effects of load $\left(F_{2,88}=3.22, p=.045, \eta_{p}^{2}=.07\right)$ and region $\left(F_{1,44}=\right.$ $\left.30.26, p<.001, \eta_{p}^{2}=.41\right)$ were significant. None of the effects involving group was significant $(p \mathrm{~s}>.27)$.

\section{Effects of n-back load on prefrontal activation}

To complement the functional connectivity analysis, mean changes in $\mathrm{HbO}$ (i.e., activation) in the PFC during the $n$-back task were examined (Table 4). A 3 (load) $\times 2$ (side) $\times 2$ (region) $\times 2$ (group) mixed ANOVA showed a significant main effect of group $\left(F_{1,44}=6.879, p=.012\right.$, $\left.\eta_{p}^{2}=.14\right)$, with the ASD group showing higher activation than the TD group during the $\mathrm{n}$ back task. Post hoc t-tests showed that higher activation in the ASD group was statistically significant only in the right medial region during the 0 -back condition $(\mathrm{p}=.012)$ and in the right lateral region during the 1 -back condition $(\mathrm{p}=.003)$; there were no differences in any ROI in the 2-back condition (ps $>.039)$.

\section{Discussion}

The present study used fNIRS to examine the effects of WM load on PFC connectivity during the number $n$-back task in children aged 8-12 with high-functioning ASD. Compared to the age-, IQ-, sex- and handedness-matched TD children, the children with ASD exhibited slower performance during the "low load" (i.e., 0-back) condition. Additionally, there were 
differential load-dependent functional connectivity changes in the lateral and medial PFC specifically in the right hemisphere but not in the left hemisphere. These findings suggest specific WM deficits and highlight the roles of WM load in altered PFC connectivity during WM processing in ASD.

Our finding of overall increased RTs during the n-back task among the children with ASD was consistent with previous observations of slower processing speed across various cognitive tasks in ASD (Goldstein, Minshew, \& Siegel, 1994; Nydén, Billstedt, Hjelmquist, \& Gillberg, 2001; Ozonoff \& Strayer, 2001). It is well known that RT is a measure of the speed of information processing (Vartanian, Martindale, \& Kwiatkowski, 2007; Vernon, 1983), which represents the maximum rate at which a cognitive operation can be executed (Kail \& Salthouse, 1994). In the present study, the children with ASD needed more time to achieve a similar performance level as the TD children, a pattern that corroborates a largescale $n$-back study with adults with ASD (Lever, Werkle-Bergner, Brandmaier, Ridderinkhof, \& Geurts, 2015). Thus, from the information-processing point of view, individuals with ASD may show a slowing in perceiving a stimulus, mapping it to a motor response, and/or executing the response while keeping up with new incoming information. However, the accuracy rates across different WM loads were not significantly different between the two groups, which was consistent with previous studies involving high-functioning autism (Koshino et al., 2008; Silk et al., 2006). This could possibly be explained by the phenomenon that high-functioning ASD individuals tend to achieve complex WM task completion by utilizing their general intellectual capacities as compensation (Barendse et al., 2013; Barendse et al., 2017).

The children with ASD, compared to TD controls, exhibited aberrant load-dependent intrahemispheric functional connectivity within the right PFC. Specifically, the changes in 
functional connectivity across various levels of WM load exhibited a reversed lateral-medial pattern. Visual inspection of the data (Figure 4a, b) showed a trend of increasing functional connectivity with increasing WM load in the right lateral PFC in the TD group, but such an increasing trend was seen in the right medial PFC in the ASD group. The right medial and lateral PFC are responsible for different cognitive processes supporting WM performance. While the medial PFC has been documented to be the hub for WM maintenance (Smith et al., 2018), the lateral PFC has been critically implicated in monitoring, which is the process of checking task performance over time for quality control and adjusting behavior (Stuss \& Alexander, 2007). Additionally, the right hemisphere has been heavily implicated in vigilance or sustained attention (Petersen \& Posner, 2012). Thus, disrupted connectivity within the right PFC may interfere with these cognitive processes and underlie the overall slowing in ASD.

There is accumulated evidence that altered neural connectivity is a hallmark characteristic of ASD. While early studies conceptualized these alterations as local overconnectivity and global underconnectivity (Just et al., 2012), this view has been challenged by more recent evidence that individuals with ASD sometimes exhibit local underconnectivity (MohammadRezazadeh et al., 2016). Our direct comparison between TD and high-functioning ASD children revealed that altered functional connectivity patterns in ASD are mediated by WM load within the right medial and lateral PFC, yet functional connectivity strength within these two ROIs were neither over- nor under-connected. Thus, consistent with the claim from a previous systematic review regarding functional connectivity in ASD (Hull et al., 2017), this study presents empirical evidence to support the view of aberrant local connectivity in ASD that is highly task-dependent. 
This study is one of the first to have applied fNIRS to clarify neural processing during WM processing in ASD. Consistent with previous fMRI studies with adults with ASD (Koshino et al., 2005, 2008), one recent study by Yeung, Lee, and Chan (2019) showed that adolescents with ASD exhibited increased right lateralization of PFC activation, which was positively associated with WM abilities, in response to increasing WM load (2-back $>0$-back) during an $n$-back task similar to the one employed in the present study. Specifically, the present study found significant medial PFC hyperactivation across WM loads in 0-back and 1-back conditions. As discussed above, given the role of the medial PFC in WM maintenance, our results could imply an overall increase in effort for WM maintenance in ASD during the $n$ back task. Interestingly, we did not observe between-group differences in the 2-back condition, which was in contrast to previous studies. The discrepancy in findings is likely due to age differences. Specifically, neural compensation in response to WM demand, especially for high WM load (i.e., 2-back) may already be in place in individuals with ASD during childhood, but the pattern shifts from medial PFC hyperactivation to altered lateralization as these individuals transition from childhood to adolescence. Taken together, the evidence suggests the potential importance of considering the developmental context when studying WM processing in ASD. To our knowledge, this study is the first to apply fNIRS to study cortical connectivity and report altered PFC connectivity during WM processing in ASD. Because fNIRS possesses adequate temporal and spatial resolution and can be readily used in a natural setting, it is a promising tool to study cortical connectivity in ASD.

While findings in the present study have shed new light on the association between WM load and neurophysiological activities in children with ASD, the following limitations should be noted. First, it focused only on the PFC. It is well known that WM is supported by distributed frontal-parietal networks (Owen et al., 2005). Thus, future work would benefit from examining the effect of WM load on functional connectivity between frontal and parietal 
regions. Second, we recruited only children with ASD. While a restricted age range had the benefit of increasing the homogeneity of the study sample, age may be an important factor that affects the behavioral and neurophysiological results, given the nonlinear differences in brain development between TD and ASD individuals across the lifespan (Courchesne, Campbell, \& Solso, 2011; Ecker, Bookheimer, \& Murphy, 2015). Finally, we recruited only boys with ASD. Future studies are needed to determine whether the present findings are generalizable to females with ASD.

\section{Conclusion}

The present study reported WM deficits and altered PFC connectivity during an $n$-back task in children with ASD. Because WM load influenced the pattern of PFC connectivity within the right but not the left PFC, this study highlights the importance of considering WM load to clarify the neural mechanisms underlying WM processing in ASD. Taken together, the literature implicates the right PFC in WM functioning in ASD (Koshino et al., 2005; Yeung et al., 2019). There is some preliminary evidence that transcranial direct current stimulation (Osório \& Brunoni, 2019) and transcranial magnetic stimulation (BarahonaCorrêa, Velosa, Chainho, Lopes, \& Oliveira-Maia, 2018) improve clinical and cognitive symptoms in individuals with ASD. Given that WM supports a variety of complex cognitive functions (Baddeley, 2012; Chun et al., 2011), future research exploring the effectiveness of neurostimulation over the right PFC to mitigate WM problems in ASD is warranted.

\section{Acknowledgments}

We would like to thank all the children and parents who participated in this study. This study was funded by The Hong Kong Polytechnic University research grant (ZE65).

\section{Conflict of Interest}


The authors have no conflicts of interest to disclose. 


\section{References}

Baddeley, A. (2012). Working memory: Theories, models, and controversies. Annual Review of Psychology, 63, 1-29.

Barahona-Corrêa, J. B., Velosa, A., Chainho, A., Lopes, R., \& Oliveira-Maia, A. J. (2018). Repetitive Transcranial Magnetic Stimulation for Treatment of Autism Spectrum Disorder: A Systematic Review and Meta-Analysis. Frontiers in Integrative Neuroscience, 12(27). doi:10.3389/fnint.2018.00027

Barendse, E. M., Hendriks, M. P., Jansen, J. F., Backes, W. H., Hofman, P. A., Thoonen, G., . . Aldenkamp, A. P. (2013). Working memory deficits in high-functioning adolescents with autism spectrum disorders: neuropsychological and neuroimaging correlates. Journal of neurodevelopmental disorders, 5(1), 14.

Barker, J. W., Aarabi, A., \& Huppert, T. J. (2013). Autoregressive model based algorithm for correcting motion and serially correlated errors in fNIRS. Biomedical optics express, 4(8), 1366-1379.

Boas, D. A., Dale, A. M., \& Franceschini, M. A. (2004). Diffuse optical imaging of brain activation: approaches to optimizing image sensitivity, resolution, and accuracy. Neuroimage, 23, S275-S288.

Chun, M. M., Golomb, J. D., \& Turk-Browne, N. B. (2011). A taxonomy of external and internal attention. Annual Review of Psychology, 62, 73-101.

Constantino, J. N., \& Gruber, C. P. (2012). Social responsiveness scale: SRS-2: Western Psychological Services Torrance, CA.

Corbett, B. A., Constantine, L. J., Hendren, R., Rocke, D., \& Ozonoff, S. (2009). Examining executive functioning in children with autism spectrum disorder, attention deficit hyperactivity disorder and typical development. Psychiatry Res, 166(2-3), 210-222. doi:10.1016/j.psychres.2008.02.005

Courchesne, E., Campbell, K., \& Solso, S. (2011). Brain growth across the life span in autism: age-specific changes in anatomical pathology. Brain Research, 1380, 138145.

Cui, J., Gao, D., Chen, Y., Zou, X., \& Wang, Y. (2010). Working memory in early-school-age children with Asperger's syndrome. J Autism Dev Disord, 40(8), 958-967. doi:10.1007/s10803-010-0943-9

Cui, X., Bray, S., Bryant, D. M., Glover, G. H., \& Reiss, A. L. (2011). A quantitative comparison of NIRS and fMRI across multiple cognitive tasks. Neuroimage, 54(4), 2808-2821.

de Vries, M., \& Geurts, H. M. (2014). Beyond individual differences: are working memory and inhibition informative specifiers within ASD? J Neural Transm (Vienna), 121(9), 1183-1198. doi:10.1007/s00702-014-1225-Z

Delpy, D. T., Cope, M., van der Zee, P., Arridge, S., Wray, S., \& Wyatt, J. (1988). Estimation of optical pathlength through tissue from direct time of flight measurement. Physics in Medicine \& Biology, 33(12), 1433.

Ecker, C., Bookheimer, S. Y., \& Murphy, D. G. M. (2015). Neuroimaging in autism spectrum disorder: brain structure and function across the lifespan. The Lancet Neurology, 14(11), 1121-1134. doi:https://doi.org/10.1016/S1474-4422(15)00050-2

Ehlis, A.-C., Bähne, C. G., Jacob, C. P., Herrmann, M. J., \& Fallgatter, A. J. (2008). Reduced lateral prefrontal activation in adult patients with attention-deficit/hyperactivity disorder (ADHD) during a working memory task: a functional near-infrared spectroscopy (fNIRS) study. Journal of psychiatric research, 42(13), 1060-1067.

Fishburn, F. A., Norr, M. E., Medvedev, A. V., \& Vaidya, C. J. (2014). Sensitivity of fNIRS to cognitive state and load. Front Hum Neurosci, 8, 76. doi:10.3389/fnhum.2014.00076 
Fletcher, P. C., \& Henson, R. N. A. (2001). Frontal lobes and human memory: insights from functional neuroimaging. Brain, 124(5), 849-881.

Frank, M. J., Loughry, B., \& O'Reilly, R. C. (2001). Interactions between frontal cortex and basal ganglia in working memory: a computational model. Cognitive, Affective, \& Behavioral Neuroscience, 1(2), 137-160.

Gabig, C. S. (2008). Verbal working memory and story retelling in school-age children with autism. Language, Speech, and Hearing Services in Schools.

Geurts, H. M., Verté, S., Oosterlaan, J., Roeyers, H., \& Sergeant, J. A. (2004). How specific are executive functioning deficits in attention deficit hyperactivity disorder and autism? Journal of child psychology and psychiatry, 45(4), 836-854.

Goldstein, G., Minshew, N. J., \& Siegel, D. J. (1994). Age differences in academic achievement in high-functioning autistic individuals. Journal of clinical and experimental neuropsychology, 16(5), 671-680.

Habib, A., Harris, L., Pollick, F., \& Melville, C. (2019). A meta-analysis of working memory in individuals with autism spectrum disorders. PLoS One, 14(4), e0216198.

Happé, F., \& Frith, U. (2006). The weak coherence account: detail-focused cognitive style in autism spectrum disorders. Journal of autism and developmental disorders, 36(1), 525.

Hull, J. V., Dokovna, L. B., Jacokes, Z. J., Torgerson, C. M., Irimia, A., \& Van Horn, J. D. (2017). Resting-state functional connectivity in autism spectrum disorders: A review. Frontiers in Psychiatry, 7, 205.

Huppert, T. J. (2016). Commentary on the statistical properties of noise and its implication on general linear models in functional near-infrared spectroscopy. Neurophotonics, 3(1), 010401.

Itahashi, T., Yamada, T., Watanabe, H., Nakamura, M., Ohta, H., Kanai, C., . . Hashimoto, R.-i. (2015). Alterations of local spontaneous brain activity and connectivity in adults with high-functioning autism spectrum disorder. Molecular autism, 6(1), 30.

Jasper, H. H. (1958). The ten-twenty electrode system of the International Federation. Electroencephalogr. Clin. Neurophysiol., 10, 370-375.

Jurcak, V., Tsuzuki, D., \& Dan, I. (2007). 10/20, 10/10, and 10/5 systems revisited: their validity as relative head-surface-based positioning systems. Neuroimage, 34(4), 16001611.

Just, M. A., Keller, T. A., Malave, V. L., Kana, R. K., \& Varma, S. (2012). Autism as a neural systems disorder: a theory of frontal-posterior underconnectivity. Neuroscience \& Biobehavioral Reviews, 36(4), 1292-1313.

Kail, R., \& Salthouse, T. A. (1994). Processing speed as a mental capacity. Acta psychologica, 86(2-3), 199-225.

Kamran, M. A., Mannan, M. M. N., \& Jeong, M. Y. (2016). Cortical signal analysis and advances in functional near-infrared spectroscopy signal: a review. Frontiers in Human Neuroscience, 10, 261.

Khan, S., Gramfort, A., Shetty, N. R., Kitzbichler, M. G., Ganesan, S., Moran, J. M., . . Joseph, R. M. (2013). Local and long-range functional connectivity is reduced in concert in autism spectrum disorders. Proceedings of the National Academy of Sciences, 110(8), 3107-3112.

Kleinhans, N., Akshoomoff, N., \& Delis, D. C. (2005). Executive functions in autism and Asperger's disorder: flexibility, fluency, and inhibition. Developmental neuropsychology, 27(3), 379-401.

Koshino, H., Carpenter, P. A., Minshew, N. J., Cherkassky, V. L., Keller, T. A., \& Just, M. A. (2005). Functional connectivity in an fMRI working memory task in high-functioning autism. Neuroimage, 24(3), 810-821. 
Koshino, H., Kana, R. K., Keller, T. A., Cherkassky, V. L., Minshew, N. J., \& Just, M. A. (2008). fMRI investigation of working memory for faces in autism: visual coding and underconnectivity with frontal areas. Cerebral cortex, 18(2), 289-300.

Lemon, J. M., Gargaro, B., Enticott, P. G., \& Rinehart, N. J. (2011). Brief report: Executive functioning in autism spectrum disorders: A gender comparison of response inhibition. Journal of autism and developmental disorders, 41(3), 352-356.

Lever, A. G., Werkle-Bergner, M., Brandmaier, A. M., Ridderinkhof, K. R., \& Geurts, H. M. (2015). Atypical working memory decline across the adult lifespan in autism spectrum disorder? J Abnorm Psychol, 124(4), 1014-1026. doi:10.1037/abn0000108

Lord, C., Rutter, M., \& Le Couteur, A. (1994). Autism Diagnostic Interview-Revised: a revised version of a diagnostic interview for caregivers of individuals with possible pervasive developmental disorders. Journal of autism and developmental disorders, 24(5), 659-685.

Ma, L., Steinberg, J. L., Hasan, K. M., Narayana, P. A., Kramer, L. A., \& Moeller, F. G. (2012). Working memory load modulation of parieto - frontal connections: Evidence from dynamic causal modeling. Human brain mapping, 33(8), 1850-1867.

Minshew, N. J., Goldstein, G., \& Siegel, D. J. (1997). Neuropsychologic functioning in autism: Profile of a complex information processing disorder. Journal of the International Neuropsychological Society, 3(4), 303-316.

Mohammad-Rezazadeh, I., Frohlich, J., Loo, S. K., \& Jeste, S. S. (2016). Brain connectivity in autism spectrum disorder. Current opinion in neurology, 29(2), 137.

Nydén, A., Billstedt, E., Hjelmquist, E., \& Gillberg, C. (2001). Neurocognitive stability in Asperger syndrome, ADHD, and reading and writing disorder: a pilot study. Developmental Medicine and Child Neurology, 43(3), 165-171.

Orihuela-Espina, F., Leff, D. R., James, D. R., Darzi, A. W., \& Yang, G.-Z. (2010). Quality control and assurance in functional near infrared spectroscopy (fNIRS) experimentation. Physics in Medicine \& Biology, 55(13), 3701.

Osório, A. A. C., \& Brunoni, A. R. (2019). Transcranial direct current stimulation in children with autism spectrum disorder: a systematic scoping review. Developmental Medicine \& Child Neurology, 61(3), 298-304. doi:10.1111/dmcn.14104

Owen, A. M., McMillan, K. M., Laird, A. R., \& Bullmore, E. (2005). N - back working memory paradigm: A meta - analysis of normative functional neuroimaging studies. Human brain mapping, 25(1), 46-59.

Ozonoff, S., \& Strayer, D. L. (2001). Further evidence of intact working memory in autism. Journal of autism and developmental disorders, 31(3), 257-263.

Petersen, S. E., \& Posner, M. I. (2012). The attention system of the human brain: 20 years after. Annual review of neuroscience, 35, 73-89.

Sanchez, C. E., Richards, J. E., \& Almli, C. R. (2012). Age-specific MRI templates for pediatric neuroimaging. Developmental neuropsychology, 37(5), 379-399.

Santosa, H., Aarabi, A., Perlman, S. B., \& Huppert, T. (2017). Characterization and correction of the false-discovery rates in resting state connectivity using functional near-infrared spectroscopy. Journal of biomedical optics, 22(5), 055002.

Santosa, H., Zhai, X., Fishburn, F., \& Huppert, T. (2018). The NIRS brain AnalyzIR toolbox. Algorithms, 11(5), 73.

Singh, A. K., Okamoto, M., Dan, H., Jurcak, V., \& Dan, I. (2005). Spatial registration of multichannel multi-subject fNIRS data to MNI space without MRI. Neuroimage, $27(4), 842-851$.

Sinzig, J., Morsch, D., Bruning, N., Schmidt, M. H., \& Lehmkuhl, G. (2008). Inhibition, flexibility, working memory and planning in autism spectrum disorders with and 
without comorbid ADHD-symptoms. Child and adolescent psychiatry and mental health, 2(1), 4.

Smith, E. E., \& Jonides, J. (1999). Storage and executive processes in the frontal lobes. Science, 283(5408), 1657-1661.

Strangman, G., Culver, J. P., Thompson, J. H., \& Boas, D. A. (2002). A quantitative comparison of simultaneous BOLD fMRI and NIRS recordings during functional brain activation. Neuroimage, 17(2), 719-731.

Stuss, D. T., \& Alexander, M. P. (2007). Is there a dysexecutive syndrome? Philosophical Transactions of the Royal Society B: Biological Sciences, 362(1481), 901-915.

Sun, J., Liu, F., Wang, H., Yang, A., Gao, C., Li, Z., \& Li, X. (2019). Connectivity properties in the prefrontal cortex during working memory: a near-infrared spectroscopy study. Journal of biomedical optics, 24(5), 051410.

Urbain, C., Vogan, V. M., Ye, A. X., Pang, E. W., Doesburg, S. M., \& Taylor, M. J. (2016). Desynchronization of fronto - temporal networks during working memory processing in autism. Human brain mapping, 37(1), 153-164.

Varshney, V., Liapounova, N., Golestani, A.-M., Goodyear, B., \& Dunn, J. (2012). Detection of inter-hemispheric functional connectivity in motor cortex with coherence analysis. Journal of the European Optical Society-Rapid publications, 7.

Vartanian, O., Martindale, C., \& Kwiatkowski, J. (2007). Creative potential, attention, and speed of information processing. Personality and Individual Differences, 43(6), 14701480.

Vernon, P. A. (1983). Speed of information processing and general intelligence. Intelligence, 7(1), 53-70.

Villringer, A., \& Chance, B. (1997). Non-invasive optical spectroscopy and imaging of human brain function. Trends in neurosciences, 20(10), 435-442.

Wechsler, D. (2003). Wechsler intelligence scale for children-Fourth Edition (WISC-IV). San Antonio, TX: The Psychological Corporation.

Williams, D. L., Goldstein, G., \& Minshew, N. J. (2006). The profile of memory function in children with autism. Neuropsychology, 20(1), 21-29. doi:10.1037/0894-4105.20.1.21

Yeung, M. K., Han, Y. M., Sze, S. L., \& Chan, A. S. (2014). Altered right frontal cortical connectivity during facial emotion recognition in children with autism spectrum disorders. Research in Autism Spectrum Disorders, 8(11), 1567-1577.

Yeung, M. K., Lee, T. L., \& Chan, A. S. (2019). Right-lateralized frontal activation underlies successful updating of verbal working memory in adolescents with high-functioning autism spectrum disorder. Biological Psychology, 148, 107743.

Yeung, M. K., Sze, S. L., Woo, J., Kwok, T., Shum, D. H. K., Yu, R., \& Chan, A. S. (2016). Reduced Frontal Activations at High Working Memory Load in Mild Cognitive Impairment: Near-Infrared Spectroscopy. Dementia and Geriatric Cognitive Disorders, 42(5-6), 278-296. doi:10.1159/000450993 


\section{Figure captions}

Figure 1: Number n-back paradigm used in this study

Figure 2: Arrangement of channels a) in the sensor space and b) in the brain space. Channels in red and blue represent the lateral and medial prefrontal cortex respectively.

Figure 3: Channel pairs for the intrahemispheric (left) and interhemispheric (right) connectivity measures in the a) lateral (red) and b) medial (blue) prefrontal cortex.

Figure 4: The effect of n-back task load on the a) left lateral, b) left medial, c) right lateral and d) right medial intrahemispheric functional connectivity changes in the prefrontal cortex in TD and ASD 
Figures

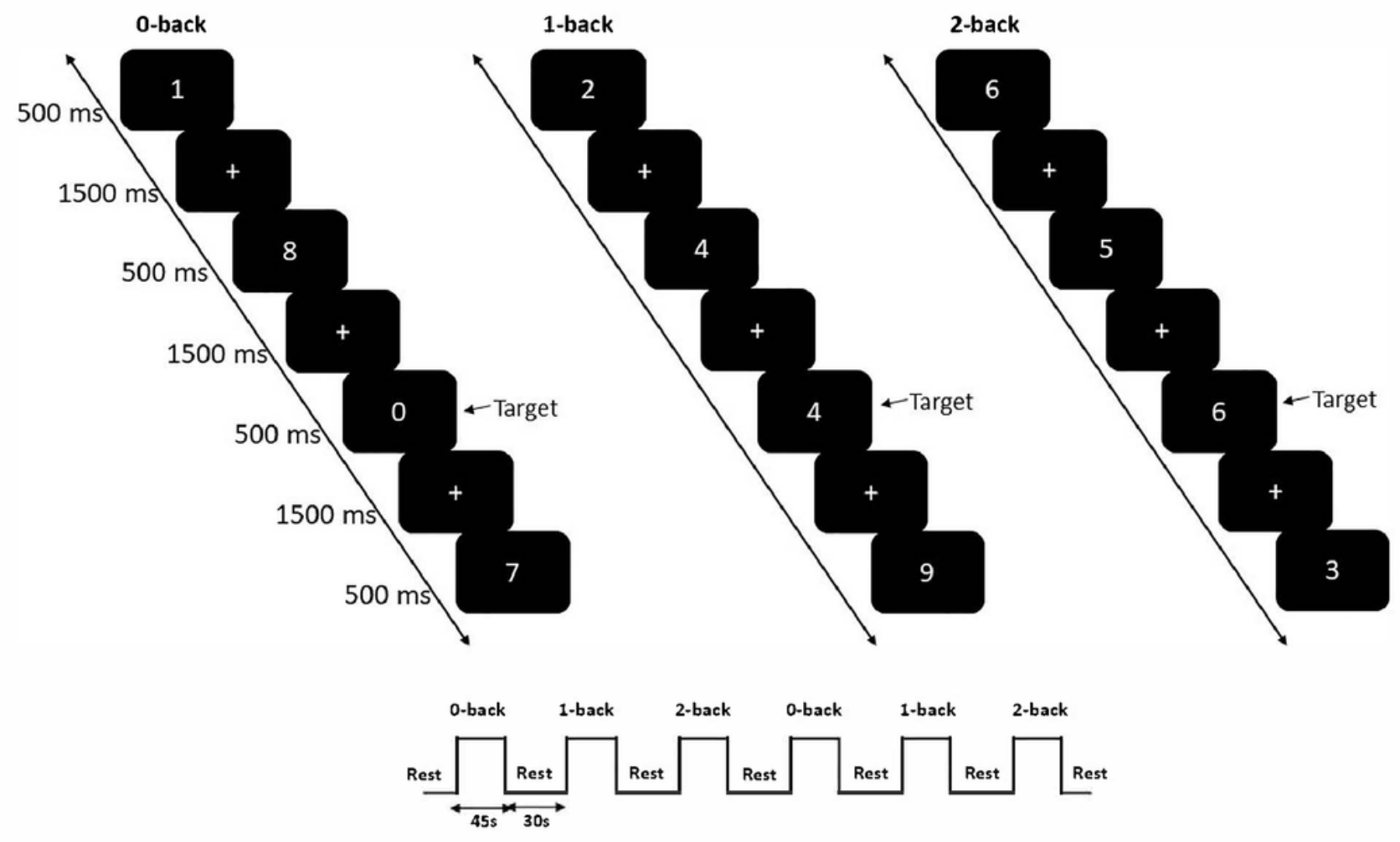

Figure 1

Number n-back paradigm used in this study 


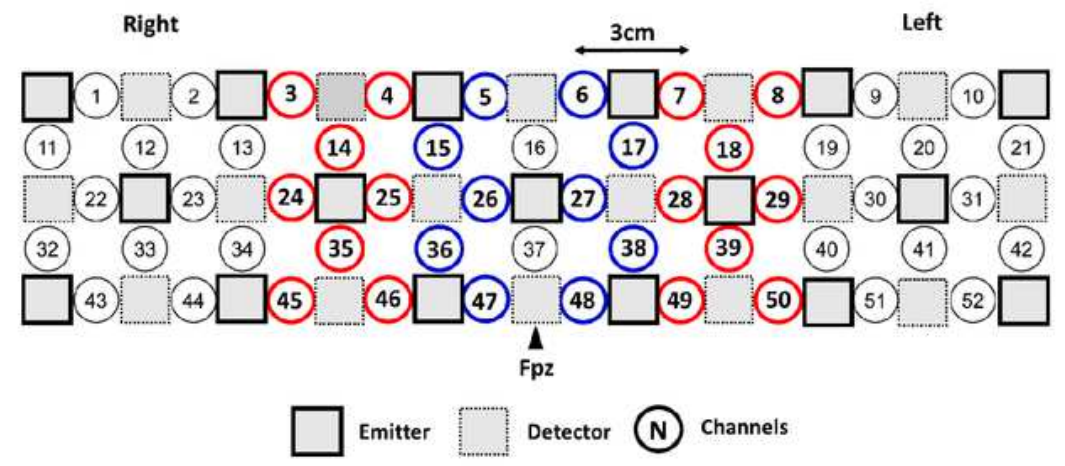

b)

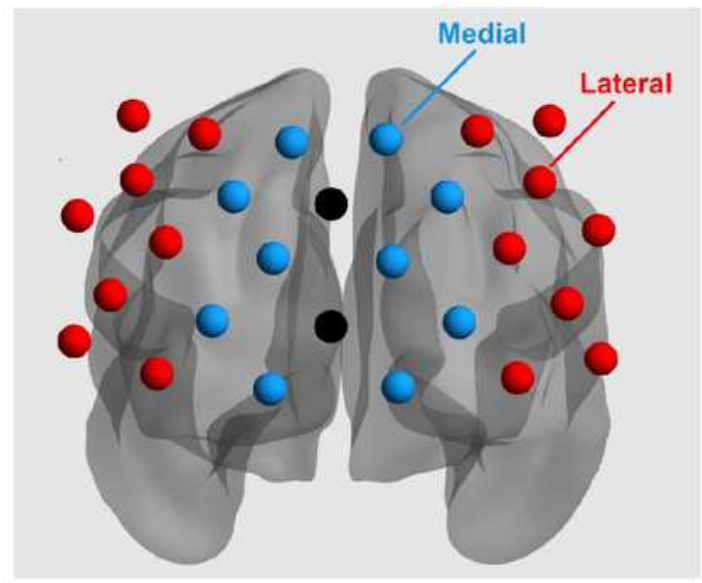

\section{Figure 2}

Arrangement of channels a) in the sensor space and b) in the brain space. Channels in red and blue represent the lateral and medial prefrontal cortex respectively. 
a)
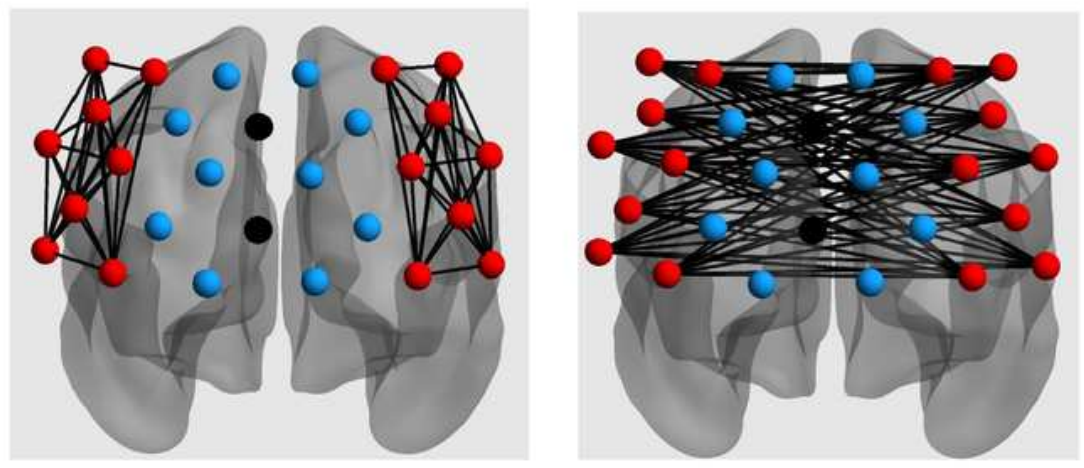

b)
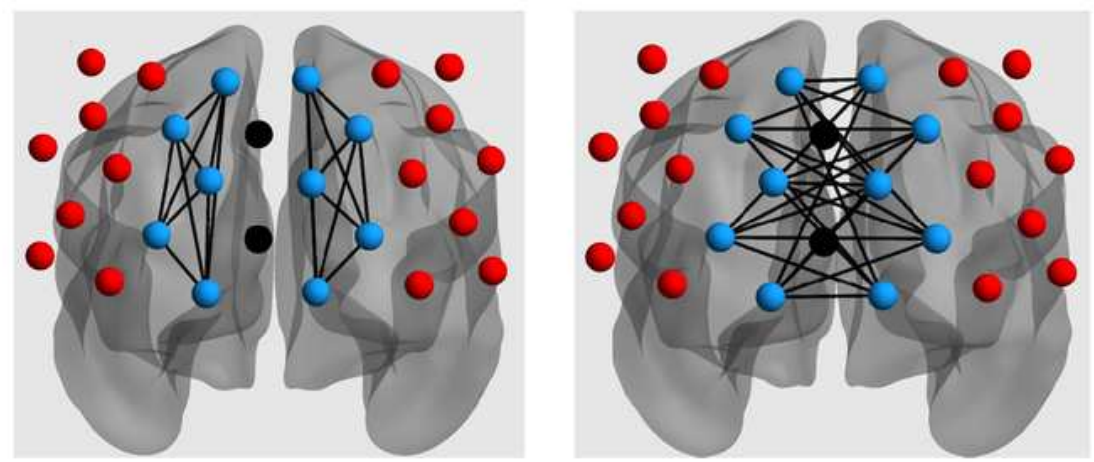

\section{Figure 3}

Channel pairs for the intrahemispheric (left) and interhemispheric (right) connectivity measures in the a) lateral (red) and b) medial (blue) prefrontal cortex. 
a)

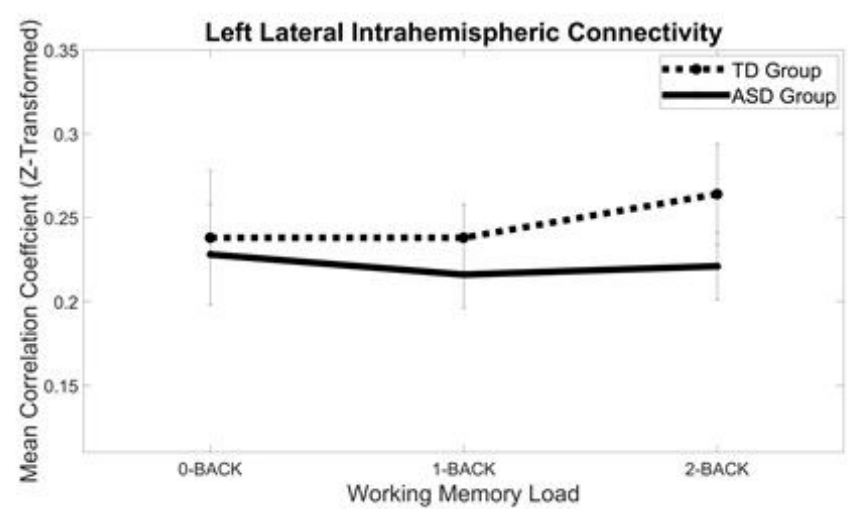

c)

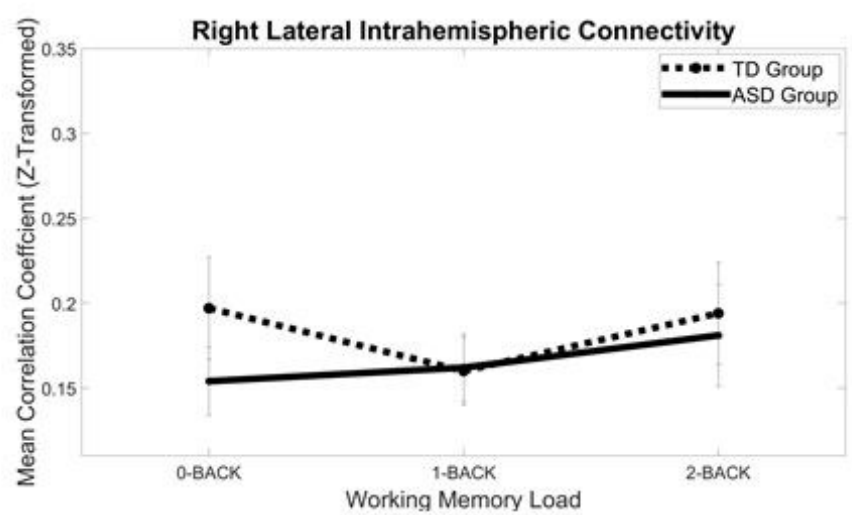

b)

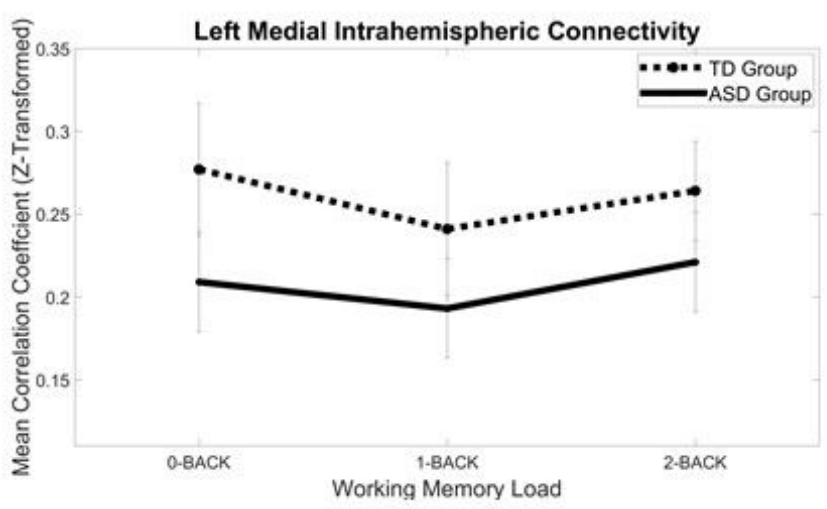

d)

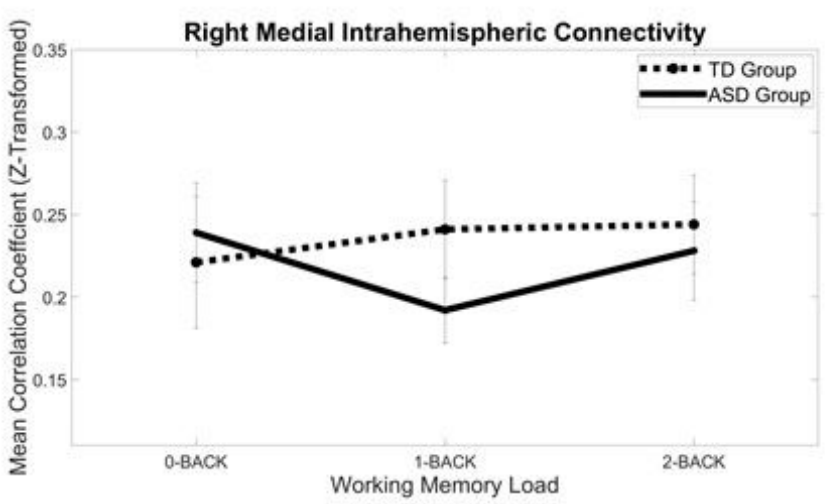

\section{Figure 4}

The effect of n-back task load on the a) left lateral, b) left medial, c) right lateral and d) right medial intrahemispheric functional connectivity changes in the prefrontal cortex in TD and ASD 